\title{
A Mobile Science Index for Development
}

\author{
http://dx.doi.org/10.3991/ijim.v6i1.1751 \\ Enrique Canessa, Marco Zennaro \\ International Centre for Theoretical Physics (ICTP), Trieste, Italy
}

\begin{abstract}
A simple Mobile Science Potentiality Index (mSPI) is introduced as a barometer reflecting a new signal of world development through the capacity for a interconnected community to participate collectively in, and carry out, scientific studies using mobile devices. The mSP Index could be a new signal for world development.
\end{abstract}

Index Terms - mobile devices, science, information technology, development

\section{INTRODUCTION}

There are billions of mobile phone subscribers today around the world out pacing fixed-line Internet users with more than thousands new users added every minute. About $60 \%$ of them live in developing countries, making mobile phones the first telecommunications technology in history to have more users there than in the developed world. For example, cell phone usage in Africa is growing almost twice as fast as any other region and jumped from 63 million users two years ago to 152 million last year [2]. Most of these countries have reported impressive adoption levels of mobile phones, a phenomenon that is now creating a paradigm shift, namely, computing and rich-media communications moving from traditional PC's to mobile devices (smart-phones, tablets, netbooks and others).

Originally, Internet and mobiles were separated technologies but now they are merging together. Mobile technology is becoming a regular part of everyone's daily lives both in the developed and developing world. Advancements in the smart-phone technology have produced such powerful gadgets and applications that can compete with PC's in this 21st century. With this kind of new computer power of mobiles, analysts and programmers can now develop scientific applications to address numerous challenges. These include helping to prevent disease outbreaks, building a census or tracking agricultural stock levels. Data gathering with mobile devices can help to save time and money for organizations while also improving information accuracy including those needed from/for developing countries. The world-wide adoption of mobile devices is opening new ways for development including, directly and for the first time, the poorest regions of the world.

Data forecasting from the International Telecommunications Union [1] indicates that in only a few years half of the planet's population will have access to the Internet through a mobile device. The possibility of connecting with mobile devices to the Internet via broadband UMTS networks at reasonable subscription prices is growing fast everywhere. This means that a majority of the world's population will soon access to the Internet via their mobile devices. While this is a proxy for actual phone and Internet usage, it is nonetheless also an indirect indicator that interest on mobiles is increasing in education, academia and scientific sectors too. Motivated by this revolution on mobile access to Internet, we discuss here a simple Mobile Science (m-Science) Potentiality Index which could reflect a new signal of world development through the capacity for a interconnected community to participate collectively in, and carry out, scientific studies using mobile devices.

\section{2. M-SCIENCE AND MSP INDEX}

Mobile Science (or "m-Science" in short) is becoming an state-of-the-art discipline because of unprecedented technological developments being done in the field of information and communications. m-Science as such comprises three main subjects of interest with a great impact on the society: Sensing, Computing and Dissemination of scientific knowledge by the use of mobile devices. These, in turn, incorporate:

- mobile data gathering by the use of sensors and mobiles,

- the analysis and process of mobile data (by mobile devices too) and

- the access to on-line services and applications directed to nurture scientists and scholars (such as mobile access to e-Journals, podcasts, web lectures and webinars, virtual conferences, mobile collaboration tools, m-Learning, etc).

The release, under a Creative Commons license, of a premier open book on m-Science by the Science Dissemination Unit (SDU) of the ICTP in trieste, Italy [5], is an effort to engage the scientific community, engineers and scholars worldwide in the design, development and deployment of the newest mobile applications. The goal is to create awareness on the huge possibilities of Mobile Science as well as to motivate a new generation of learners, scholars and scientists. The book gives a balanced mix of technical detail, general overview, societal impact and a sense of the possible.

We devise here an approximated Mobile Science Potentiality Index (mSPI) to be used as a barometer reflecting the potential and capacity for a community (for example a group of researchers) in a nation or region to develop and participate in m-Science. The mSPI we introduce is based on a simple relation:

"The ratio of the mobile broadband subscriptions available by country and the amount of researchers in labor force (working full time in different fields of science) in a country". For illustrative purposes, an example of how the mSP Index may behave on a nation wide scale follows in Table I by using recent published data based on the OECD's definition of wireless broadband data (2010). The broadband subscribers per 100 inhabitants (2009) 
PAPER

A Mobile SCIENCE INDEX FOR DEVELOPMENT

includes satellite, terrestrial fixed wireless, dedicated and standard mobiles, which certainly gives a crude estimation for mobile access to Internet in the sense given within mScience, but it can be a useful ratio to obtain a reasonable indication of the current potentiality of m-Science in some places of the industrialized world where discrete data is only available so far. Hopefully, data for developing countries will be made available with the increasing usage of mobile devices and the participation of these countries in the internalization of science.

\section{REMARKS}

From Table I it can be deduced that when communities have greater access to broadband facilities, then researchers (even if fewer than other work forces in a country) and broadband subscribers can participate in m-Science experiments leading to a greater mSP Index. In a thought experiment, this mSPI index may in principle score and monitor over time, a community (consisting of "not only" researchers) on a scale from 1 (fully mobile and connected community) to 0 (highly localized and isolated community). As m-Science through mobile sensing, computing and dissemination continues to become less and less expensive, more integrated and more ubiquitous, the hope is that projects in this new field will increasingly be within reach of scientists in the less industrialized world too. A world-wide mobile accessibility will help bootstrap participatory and truly societal scale networks for science research. An increasing mSPI measure could well be a useful, reliable instrument to attempt quantifying the world-wide expansion of, and interest on, the new grow- ing $\mathrm{m}$-Science discipline in years to come depicting a rapidly changing landscape.

The mobility of the phone provides some other important scientific and economical opportunities. Data collection with a mobile phone has the potential to dramatically improve services that relies on accurate and up-to-date information. The more time-critical the information, and the more remote the location, the more scientists have to gain from a mobile phone based solution. Thus, mScience offers tremendous benefits to academic research and to society as a whole and the mSP Index could be a new signal for world development. It is surprising to note that the maximum reported mSPI value of $74,20 \%$ is for the Netherlands and the minimum is $25.57 \%$ for Japan, contrary to what it would it be expected.

Based on information extracted from the Web of Science (Thomson Reuters) database of scientific publications spanning from 1980 to 2009, the world's scientific production has grown from about 400,000 to $1,200,000$ publications in the last three decades. This increment of interest in science, together with the recent technological developments in mobile technologies, is helping to make of the new m-Science a completely new research field. The potential for m-Science and science-related mobile apps, is unlimited. Since the majority of new generation devices can access the web in some way, mobile technological tools can be used to collect basic information as well as to exchange information and to access scientific computing among other services and carrying lots of benefits that permeates into society.

TABLE I.

MoBILE SCIENCE POTENTIALITY INDEX (MSPI) ${ }^{1}$

\begin{tabular}{|c|c|c|c|}
\hline Country & $\begin{array}{c}\text { Researchers in labor force } \\
\text { per 10000 inhabitants (1999) }\end{array}$ & $\begin{array}{c}\text { Broadband subscribers } \\
\text { per 100 inhabitants (2009) }\end{array}$ & mSPI (\%) \\
\hline Netherlands & 50 & 37.1 & $\mathbf{7 4 . 2 0}$ \\
\hline Austria & 34 & 22.1 & $\mathbf{6 5 . 0 0}$ \\
\hline Switzerland & 55 & 35.6 & $\mathbf{6 2 . 1 2}$ \\
\hline Italy & 33 & 20.5 & $\mathbf{6 0 . 8 2}$ \\
\hline Denmark & 61 & 37.1 & $\mathbf{5 3 . 7 0}$ \\
\hline Belgium & 54 & 29.0 & $\mathbf{5 3 . 6 3}$ \\
\hline United Kingdom & 55 & 29.5 & $\mathbf{5 2 . 7 3}$ \\
\hline New Zealand & 44 & 23.2 & $\mathbf{5 1 . 0 3}$ \\
\hline Canada & 58 & 29.6 & $\mathbf{5 0 . 5 0}$ \\
\hline Germany & 60 & 30.3 & $\mathbf{4 9 . 8 4}$ \\
\hline France & 61 & 30.4 & $\mathbf{4 3 . 4 6}$ \\
\hline Norway & 78 & 33.9 & $\mathbf{3 8 . 2 4}$ \\
\hline Ireland & 51 & 19.5 & $\mathbf{3 5 . 6 0}$ \\
\hline Sweden & 91 & 32.4 & $\mathbf{3 2 . 5 9}$ \\
\hline USA & 81 & 26.4 & $\mathbf{3 4 . 7 8}$ \\
\hline Australia & 67 & 23.3 & $\mathbf{2 6 . 9 7}$ \\
\hline Finland & 99 & 26.7 & $\mathbf{2 5 . 5 7}$ \\
\hline Japan & 97 & 24.8 & \\
\hline
\end{tabular}

1 Data from OECD: http://www.oecd.org/ 
PAPER

A MOBILE SCIENCE INDEX FOR DEVELOPMENT

Mobile technology can be used in powerful new ways to carry out scientific research, analyze and share results to many. Mobile phones are, for instance, being used to gather scientific data from remote and isolated places that would be impossible to retrieve by other means. Scientists, acting alone or in groups, are starting to use mobile devices and web-based mobile applications to systematically explore interesting scientific aspects of their surroundings ranging from climate change to earthquake monitoring world-wide. Accurate and timely field data gathering is possible with mobile phones. By attaching sensors to GPS-enabled cell phones, it is possible to gather the raw data necessary to begin to understand how, for example, urban air pollution impacts both individuals and communities. While integrating a sensor into a phone and transmitting the data that it gathers to a database is posssible, doing so at low cost, on a societal scale, with millions of phones providing data from hundreds of networks spread throughout the world makes this possibility a challenging one. This is a unique m-Science occasion that deserves further attention and promotion world-wide (Canessa et al. 2009). At the expense of sampling a given location continuously, a sensor in a user's smart-phone can provide significant geographic coverage. Also, mobile sensors will be heavily biased towards locations in which people congregate, so for human-centric applications, sensing in mobile devices will often provide coverage exactly where it is needed most. In over-sampled locations, the precision of the sensing system can be increased by carefully averaging the readings from several nearby sensors.

All these advantages point to build the largest scientific instrument ever built, consisting of millions or billions of sensors (attached in some cases to mobiles), aggregating data on an unprecedented scale. This instrument could be truly societal scale, reaching across economic, social, geographic and political boundaries, and illuminating the corners of human activity, how our environment affects us, and how we affect our environment. Besides, supercomputing on a cellphone using this huge data will become also a reality.

The development of scientific mobile applications need certainly still to be simplified without the needs for having high computer literacy. There is a useful and simple blocks language for mobile phones as for example "App Inventor for Android" [6]. This is a visual programming environment for building mobile apps. by putting together blocks that represent the phone's functionality so, even those with no programming experience can use App Inventor to create mobile apps. On the other hand, mobile application development with the alternative Python (better known as "Python on Symbian or PyS60") is also a powerful simple code to build/prototype mobile applica- tions rapidly with interface for telephony, messaging, multimedia, camera, sensors and location tracking.

The wide availability of cellular telephones equipped with cameras opens many other opportunities for inexpensive, portable photometric measurements suitable for educational purposes. Spectrophotometry can make more sense to students around the world when they can see light change intensity when passed through a sample than when just seen equations, sketches or the output of a computer screen or meter. Mobile social networks are rapidly being organized by young people for the dissemination and credibility of information. Besides, m-Science technology through Internet access opens up also amazing educational opportunities. With m-Learning, students can also take advantage as learning is now freed from the restrictions of location. Mobile learning programs can specially foster Physics and Mathematics m-Learning.

All of this novel m-Science activity aims to engage the scientific community, engineers and scholars world-wide in the design, development, deployment and usage of the newest mobile applications which benefits many and giving a substantial contribution to the world development. The proposed Mobile Science Potentiality Index can be used to best assess a country's development in science and mobile technology innovation and as a barometer reflecting a new signal of world development through the capacity for a interconnected community to participate collectively in, and carry out, scientific studies using mobile devices.

\section{REFERENCES}

[1] ITU - International Telecommunications Union, (2010) $<$ http://www.itu.int $>$

[2] EPROM - Entrepreneurial Programming and Research on Mobiles of the MIT- Massachusetts Institute of Technology, (2009) $<$ http://eprom.mit.edu/whyafrica.html $>$.

[3] OECD - Organisation for Economic Co-operation and Development, (2010) <http://www.oecd.org >

[4] E. Canessa, C. Fonda and M. Zennaro, Supporting Science in Developing Countries using Open Technologies (2009) Eur. J. Phys. 30, 651-660. http://dx.doi.org/10.1088/0143-0807/30/3/022

[5] E. Canessa and M. Zennaro (Eds.), m-Science: Sensing, Computing and Dissemination, ISBN 92-95003-43-8 (2010) $<$ http://www.m-science.net/book/>

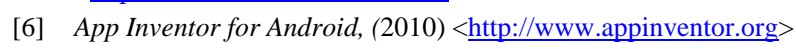

\section{AUTHORS}

Enrique Canessa and Marco Zennaro are with Science Dissemination Unit (SDU), International Centre for Theoretical Physics (ICTP), Trieste, Italy.

Received 14 July 2011. Published as resubmitted by the authors 14 December 2011. 